\title{
Migration from a Mitogenic Niche Promotes Cell-Cycle Exit
}

\author{
Yoojin Choi, ${ }^{1,2}$ Paul R. Borghesani, ${ }^{1,2}$ Jennifer A. Chan, ${ }^{1,3}$ and Rosalind A. Segal ${ }^{1,2}$ \\ ${ }^{1}$ Department of Pediatric Oncology, Dana-Farber Cancer Institute, ${ }^{2}$ Department of Neurobiology, Harvard Medical School, and ${ }^{3}$ Department of Pathology, \\ Brigham and Women's Hospital, Boston, Massachusetts 02115
}

\begin{abstract}
During development, neural precursors proliferate in one location and migrate to the residence of their mature function. The transition from a proliferative stage to a migratory stage is a critical juncture; errors in this process may result in tumor formation, mental retardation, or epilepsy. This transition could be the result of a simple sequential process in which precursors exit the cell cycle and then begin to migrate or a dynamically regulated process in which migration away from a mitogenic niche induces precursors to exit the cell cycle. Here, we show, using in vivo and in vitro approaches, that granule cell precursors proliferate when they are exposed to the microenvironment of the external granule cell layer (EGL) and exit the cell cycle as a result of migrating away from this environment. In vivo, granule cell precursors that remain in the EGL because of impaired migration continue to proliferate in the mitogenic niche of the EGL. In vitro, granule cell precursors that are introduced into an organotypic cerebellar slice proliferate preferentially in the EGL. We identify Sonic Hedgehog as a critical component of the EGL mitogenic niche. Together, these data indicate that migration away from a mitogenic niche promotes transition from a proliferative to a nonproliferative, migratory stage.
\end{abstract}

Key words: proliferation; BDNF; cerebellum; granule cells; Sonic Hedgehog; microenvironment

\section{Introduction}

During neural development, precursor cells proliferate in spatially restricted zones before migrating to the residence of their mature function. Thus, a critical juncture in development is the transition from a proliferative to a migratory stage. Diseases associated with failure of this process include brain tumors, mental retardation, and epilepsy (Flint and Kriegstein, 1997; Gleeson, 2000; Kim et al., 2003; Im et al., 2004). Two mechanisms, operating separately or together, could coordinate this transition. First, precursors may stop proliferating and then sense motility cues and begin to migrate. Another possibility is that migration away from a mitogenic niche induces cell-cycle exit.

The cerebellum is an attractive system for studying this transition, because cerebellar granule cells undergo alternating phases of proliferation and migration (Altman and Bayer, 1997). During embryogenesis, cells born in the rhombic lip migrate over the nascent cerebellum to form the external granule cell layer (EGL). Granule cell precursors proliferate extensively in the outer EGL during the early postnatal period and migrate along radial glia into the internal granule cell layer (IGL). The EGL is a transient layer, disappearing in mice by 3 weeks after birth. Although it is

Received April 20, 2005; revised Sept. 27, 2005; accepted Sept. 28, 2005.

This work was supported by grants from the Pediatric Brain Tumor Foundation, by the Mahoney Foundation, and by National Institutes of Health Grant NS 37757 (R.A.S.). We thank Dr. L.-H. Tsai for p35-/ - mice, Dr. P. Silver for GFP antibody, Curis for lipid-modified SHH protein, AnorMED for AMD 3100, Dr. Q. Ma for Gli1 in situ probe, and Dr. D. H. Rowitch for Ptc in situ probe. We thank Drs. J. B. Rubin, C. Stiles, A. M. Kenney, and K. J. Nazemi for critical reading of this manuscript and for helpful discussions. We also thank Erin Berry and Maria Pazyra for technical assistance.

Correspondence should be addressed to Rosalind A. Segal, Department of Pediatric Oncology, Dana 620, DanaFarber Cancer Institute, 44 Binney Street, Boston, MA 02115. E-mail: rosalind_segal@dfci.harvard.edu.

P. R. Borghesani's present address: Department of Psychiatry and Behavioral Sciences, University of Washington Medical Center, Seattle, WA 98105.

DOI:10.1523/JNEUROSCI.1559-05.2005

Copyright $\odot 2005$ Society for Neuroscience $\quad$ 0270-6474/05/2510437-09\$15.00/0 clear that proliferation of precursors occurs exclusively in the EGL, it is not known whether the EGL constitutes a mitogenic niche, a spatially restricted environment containing extracellular cues that promote proliferation. If so, active migration away from the EGL may induce cell-cycle exit by moving the precursors out of a mitogenic environment.

Stimuli known to induce granule cell precursor proliferation include cell-cell contacts, extracellular matrix (ECM) components, and soluble growth factors (Dahmane and Ruiz-i-Altaba, 1999; Wallace, 1999; Wechsler-Reya and Scott, 1999; Pons et al., 2001; Solecki et al., 2001; Rubin et al., 2002). Sonic Hedgehog (SHH), produced by Purkinje cells, is the most efficacious mitogen known, and inhibition of $\mathrm{SHH}$ signaling reduces granule cell proliferation (Waschek et al., 1998; Dahmane and Ruiz-i-Altaba, 1999; Wallace, 1999; Wechsler-Reya and Scott, 1999; Nicot et al., 2002; Lewis et al., 2004). Thus, SHH is a potential component of a cerebellar mitogenic niche, but it is not known whether or how SHH delineates a mitogenic niche.

Here, we use in vivo and in vitro approaches to determine whether the EGL constitutes a cerebellar mitogenic niche. When granule cell precursors are forced to remain in the EGL in vivo because of a mutation that impairs migration out of the EGL, the precursors continue to proliferate. We developed an assay to test directly whether this persistent proliferation is attributable to the effect of a mitogenic niche. In this assay, we introduce a homogeneous population of granule cell precursors onto an organotypic cerebellar slice. Although the introduced precursors incorporate randomly into all layers, they proliferate preferentially in the outer EGL. The preferential proliferation arises from mitogenic factors in the EGL microenvironment, and we find that endogenous $\mathrm{SHH}$ is a critical component of this mitogenic niche. Together, these data indicate that the EGL constitutes a cerebellar mitogenic niche and that migration away from this mitogenic 
niche promotes the transition from a proliferative to a migratory stage.

\section{Materials and Methods}

Animals. Breeding pairs of brain-derived neurotrophic factor (BDNF) $+/-$ (Ernfors et al., 1994) mice were purchased from The Jackson Laboratory (Bar Harbor, ME). $p 35-/-$ mice were a generous gift from Dr. L.-H. Tsai (Harvard Medical School). Green fluorescent protein (GFP) transgenic mice were purchased from The Jackson Laboratory. The C57BL/6J background transgenic mouse line expresses enhanced GFP under the control of a $\beta$-actin promoter and cytomegalovirus enhancer. $\mathrm{BALB} / \mathrm{c}$ mouse breeding pairs were purchased from Charles River Laboratories (Wilmington, MA).

All experimental procedures were performed in accordance with the National Institutes of Health guidelines and were approved by the DanaFarber Cancer Institutional Animal Care and Use Committee.

In vivo 5-bromo-2'-deoxyuridine labeling and immunohistochemistry. In vivo 5-bromo- $2^{\prime}$-deoxyuridine (BrdU) labeling and immunohistochemistry were performed as described previously (Borghesani et al., 2002). Briefly, pups were injected with $50 \mathrm{mg} / \mathrm{kg}$ BrdU (Sigma, St. Louis, MO) $6 \mathrm{~h}$ before being killed. Sagittal cryosections $(14 \mu \mathrm{m})$ were immunostained with BrdU antibody (Roche Diagnostics, Indianapolis, IN) and visualized using cyanine 3-conjugated IgG (Jackson ImmunoResearch, West Grove, PA). Labeled cells were counted in nonadjacent midsagittal sections in two regions of the cerebellum: on folium 6 in the primary fissure and on folium 9 in the secondary fissure. Counting was done in $100 \times 100 \mu \mathrm{m}$ fields, three fields per folium per mouse. The width of the field was determined by starting at the middle of the folium along the axis of the pial surface and measuring $50 \mu \mathrm{m}$ to the left and 50 $\mu \mathrm{m}$ to the right; the depth of the area was determined by measuring 100 $\mu \mathrm{m}$ into the section. Wild-type and BDNF-/ - littermates or wild-type and $\mathrm{p} 35-/-$ littermates were processed in parallel at postnatal day 7 (P7), P14, or P17.

Organotypic slice culture. Slices were taken and cultured following the methods of Stoppini et al. (1991) and Polleux et al. (1998). Briefly, cerebella were dissected from P6 wild-type C57BL/6J pups and washed in calcium- and magnesium-free HBSS with $36 \mathrm{~mm}$ glucose and $15 \mathrm{~mm}$ HEPES, pH 7.4. Cerebella were embedded in $2 \%$ low melting-point agarose in HBSS, and $250-\mu \mathrm{m}$-thick midsagittal slices were cut in an ice-cold HBSS bath using a vibrating microtome (VT1000S; Leica, Wetzlar, Germany). After removing the agarose gel, slices were transferred to a porous membrane insert (Millipore, Billerica, MA) with culture medium containing DMEM/Ham's F-12 with N2 supplement (Invitrogen, Carlsbad, CA), $20 \mathrm{~mm} \mathrm{KCl,} 36 \mathrm{~mm}$ glucose, and penicillin-streptomycin. Slices were incubated at $37^{\circ} \mathrm{C}$ in $7.5 \% \mathrm{CO}_{2}$.

Purification and introduction of GFP + cells. A slice overlay assay was modified from Polleux and Ghosh (Polleux et al., 1998; Polleux and Ghosh, 2002). Cerebella were dissected from P6 GFP+ pups, washed in HBSS, and treated with $1 \mathrm{mg} / \mathrm{ml}$ trypsin with $125 \mathrm{U} / \mathrm{ml}$ DNase (Sigma) for $20 \mathrm{~min}$. Enzymatic digestion was quenched with $10 \%$ fetal calf serum in DMEM, and the tissue was pelleted in a clinical centrifuge. Pellets were washed in HBSS and dissociated by serial trituration. The dissociated cells were plated on a tissue culture dish coated with poly-D-lysine (50 $\mu \mathrm{g} / \mathrm{ml}$ ) for $30 \mathrm{~min}$ to allow glial cells to adhere. Nonadherent cells, now enriched for granule cell precursors, were collected, passed through a 100 $\mu \mathrm{m}$ nylon mesh cell strainer (BD Biosciences, Franklin Lakes, NJ), and counted. GFP + granule cell precursors in single-cell suspension at $1 \times$ $10^{5} \mathrm{cells} / \mathrm{ml}$ were added to the slices, $100 \mu \mathrm{l}(10,000$ cells $)$ per insert. The system was cultured for $18 \mathrm{~h}$, with $\operatorname{BrdU}(30 \mu \mathrm{g} / \mathrm{ml})$ during the last $6 \mathrm{~h}$. We verified, using immunocytochemistry, that the purified cells are indeed granule cell precursors: $94 \%$ express the granule cell-specific marker Zic (Borghesani et al., 2002), whereas 0.5\% express the astrocytic cell marker GFAP (Sigma), and 0.5\% express the Purkinje cell marker calbindin (Sigma).

Aliquots of GFP + granule cell precursors at $2 \times 10^{6}$ cells $/ \mathrm{ml}$ and $1 \times$ $10^{5}$ cells $/ \mathrm{ml}$ were plated on poly-ornithine-coated CultureWell chambered coverglass (Grace Bio-Labs, Bend, OR). These high- and lowconcentration dissociated cultures were also incubated for $18 \mathrm{~h}$ at $37^{\circ} \mathrm{C}$ in 7.5\% $\mathrm{CO}_{2}$ and labeled with $\mathrm{BrdU}$ during the last $6 \mathrm{~h}$.
Reagents. Lipid-modified $\mathrm{SHH}$ protein was a generous gift from Curis (Cambridge, MA) and used at $3 \mu \mathrm{g} / \mathrm{ml}$. Cyclopamine was purchased from Toronto Research Chemicals (North York, Ontario, Canada) and used at $10 \mu \mathrm{M}$, with DMSO as vehicle control. AMD3100 octahydrochloride (AMD 3100) was a generous gift from AnorMED (Langley, British Columbia, Canada) or was purchased from Sigma and used at $1.5 \mathrm{ng} / \mathrm{ml}$. For in situ hybridization, Gli1 probe was a generous gift from Dr. Q. Ma (Dana-Farber Cancer Institute), and Patched 1 (Ptc1) probe was a generous gift from Dr. D. H. Rowitch (Dana-Farber Cancer Institute). Hedgehog-interacting protein 1 (Hip1) probe was purchased from American Type Culture Collection (Manassas, VA).

Immunofluorescence staining of slices and parallel cultures. After $18 \mathrm{~h}$ in culture, slices with introduced GFP + cells were fixed in $4 \%$ paraformaldehyde for $30 \mathrm{~min}$ at room temperature. After thorough washing in PBS, slices were incubated in blocking solution containing $5 \%$ normal goat serum and $0.4 \%$ Triton X-100 in PBS for $1 \mathrm{~h}$ at room temperature. A rabbit polyclonal GFP antibody, a generous gift from Dr. P. Silver (DanaFarber Cancer Institute), was used at 1:6000 in 5\% normal goat serum overnight at $4^{\circ} \mathrm{C}$. Slices were washed and incubated with Alexa 488conjugated anti-rabbit IgG (Molecular Probes, Eugene, OR) for $1 \mathrm{~h}$ at room temperature. Slices were washed and treated with $2 \mathrm{~N} \mathrm{HCl}$ at $37^{\circ} \mathrm{C}$ for $30 \mathrm{~min}$, followed by two $10 \mathrm{~min}$ washes in $0.1 \mathrm{M}$ sodium borate, and rinsed in PBS. Slices were incubated in blocking solution for $30 \mathrm{~min}$ and incubated in BrdU antibody (BD Biosciences) at 1:75 in 5\% normal goat serum for $1 \mathrm{~h}$ at room temperature. Slices were washed, incubated with Alexa 546-conjugated anti-mouse IgG (Molecular Probes), and washed and mounted on microscope slides in ImmuMount (Immunotech, Marseille, France).

Dissociated cultures were treated the same way as slices, with decreased times for fixation and $\mathrm{HCl}$ treatment $(10 \mathrm{~min}$ each). Cover glasses were mounted in Vectashield mounting medium (Vector Laboratories, Burlingame, CA). BrdU+ and GFP+ cells were counted in 10 fields per cover glass. In both slices and dissociated cultures, the proliferative index was calculated by dividing the number of GFP-BrdU double-positive cells by the number of GFP + cells.

SHH immunohistochemistry. Brains were dissected from P6 C57BL/6J pups and $5-\mu \mathrm{m}$-thick cryosections taken as described above. Sections were incubated in blocking solution containing $5 \%$ heat-inactivated horse serum for $1 \mathrm{~h}$ at room temperature and incubated with goat polyclonal SHH antibody (Santa Cruz Biotechnology, Santa Cruz, CA) at 1:50 in blocking solution for $1 \mathrm{~h}$ at room temperature. Sections were washed and incubated in monoclonal calbindin antibody (Sigma) at 1:500 in blocking solution for $1 \mathrm{~h}$ at room temperature. Sections were washed, incubated with Alexa 546-conjugated anti-goat IgG and Alexa 488-conjugated anti-mouse IgG (Molecular Probes) in blocking solution for $1 \mathrm{~h}$ at room temperature, and washed three times in PBS and mounted.

In situ hybridization. In situ hybridization was performed as described previously (Rubin et al., 2002). Briefly, brains from P6 BALB/c mice were removed and fixed in $4 \%$ paraformaldehyde for $24 \mathrm{~h}$ and cryoprotected in $30 \%$ sucrose. Sagittal sections ( $14 \mu \mathrm{m}$ thick) were obtained and treated with $20 \mu \mathrm{g} / \mathrm{ml}$ proteinase $\mathrm{K}$ for $10 \mathrm{~min}$ at room temperature. Sections were fixed in paraformaldehyde and washed in PBS. Hybridization was performed with digoxigenin (DIG)-labeled sense and antisense RNA probes for $20 \mathrm{~h}$ at $65^{\circ} \mathrm{C}$ in hybridization buffer [50\% formamide, $5 \%$ SSC, $100 \mu \mathrm{g} / \mathrm{ml}$ yeast tRNA, $100 \mu \mathrm{g} / \mathrm{ml}$ heparin, $1 \%$ Denhardt's, $0.1 \%$ Tween 20,0.1\% CHAPS (3-[(3-cholamidopropyl)dimethylammonio]-1propanesulfonate), and $5 \mathrm{~mm}$ EDTA]. Sections were washed with $0.2 \%$ SSC and $0.1 \%$ Tween 20 at $65^{\circ} \mathrm{C}$. Hybridized DIG-labeled probes were visualized with an antibody to DIG and NBT/BCIP.

Microscopy. Fluorescent and bright-field images were obtained using a CCD camera on a Nikon (Tokyo, Japan) Eclipse microscope and processed with Spot Camera software (Diagnostic Instruments, Sterling Heights, MI). High-magnification fluorescent images of introduced GFP + and GFP-BrdU double-positive cells were obtained using DeltaVision (Applied Precision, Issaquah, WA) restoration fluorescence microscopy, performed on an Olympus (Tokyo, Japan) fluorescence microscope configured with a DeltaVision stage and software (Applied Precision). To confirm colocalization of staining, $Z$-series $(0.2-\mu \mathrm{m}$-thick 
serial optical sections) were acquired, and SoftWoRx imaging software (Applied Precision) was used to perform a blind deconvolution.

\section{Results}

\section{Impaired migration results in persistent proliferation in} the EGL

During development, granule cell precursors proliferate in the EGL and migrate into the IGL. To address the relationship between proliferation and migration, we first asked whether granule cell precursors continue to proliferate when they are unable to migrate out of the EGL. We showed previously that mice that lack BDNF exhibit a persistent EGL resulting from impaired granule cell migration out of the EGL (Schwartz et al., 1997; Borghesani et al., 2002). Therefore, we asked whether granule cell proliferation is altered in $B D N F-/-$ mice. We label actively dividing precursors by systemic injection with BrdU at P7, P14, or P17, and cerebella are processed and immunostained for BrdU. Proliferative index is calculated by dividing the number of BrdU + cells by the number of $4^{\prime}, 6^{\prime}$-diamidino-2-phenylindole dihydrochloride (DAPI)-labeled cells, and $B D N F-/-$ mice are compared with age-matched wild-type mice. At $\mathrm{P} 7$, the rate of proliferation in $B D N F-/-E G L$ is equal to the rate of proliferation in wild-type EGL (Fig. $1 B$ ). However, at P14, proliferative index of granule cell precursors in $B D N F-/-$ EGL is 2.7 -fold higher than in wildtype EGL (Fig. 1 $A, B$ ). The increase in proliferation persists at $\mathrm{P} 17$, showing a 1.9-fold difference. Thus, when impaired migration causes granule cell precursors to remain in the EGL in vivo, the precursors continue to proliferate.

Although persistent granule cell proliferation in $B D N F-/-$ mice could result from an inability to migrate away from a mitogenic niche in the EGL, alternatively, this result could arise if BDNF directly induces cell-cycle exit. To determine whether BDNF directly affects proliferation, we culture granule cell precursors from wild-type, $B D N F+/-$, and $B D N F-/-$ mice. The three genotypes do not differ in the basal rate of proliferation (Fig. 1C), consistent with previous studies showing that BDNF does not directly affect granule cell proliferation (Segal et al., 1992; Gao et al., 1995; Tanaka et al., 2000). Furthermore, the rate of $\mathrm{SHH}$-induced proliferation of granule cell precursors from wild-type, $B D N F+/-$, and $B D N F-/-$ mice do not differ, and exogenous $\mathrm{BDNF}$ does not alter the extent of $\mathrm{SHH}$-induced proliferation (Fig. 1C). It is possible that loss of BDNF results in increased proliferation by upregulating the expression of Shh in the cerebellum. However, in $B D N F+/-$ mice whose BDNF protein level is reduced to $50 \%$ of wild type, we detect no evidence of change in Shh mRNA level in the cerebellum compared with the wild type (data not shown). These data indicate that the persistent proliferation in the EGL of the $B D N F-/-$ mouse is not attributable to a direct effect of BDNF on granule cell precursor proliferation.

If the processes of proliferation and migration are linked in such a way that impaired migration inevitably leads to persistent proliferation, this may explain the persistent proliferation observed in the BDNF-/-EGL. To test this possibility, we analyzed granule cell proliferation in a different mutant with impaired migration, the $p 35-/-$ mouse (Tsai et al., 1994). A mutation in p35, a neuron-specific activator of Cdk5, results in impaired migration in cerebral and cerebellar cortex (Chae et al., 1997; Kwon and Tsai, 1998). In the $p 35-/-$ cerebellum, aberrantly located granule cells are detected in the molecular layer (ML) (Fig. 2A). By using BrdU to label dividing precursors in the EGL and by counting how many labeled cells are found in the EGL, ML, or IGL after $96 \mathrm{~h}$ after BrdU injection, we confirm that migration is
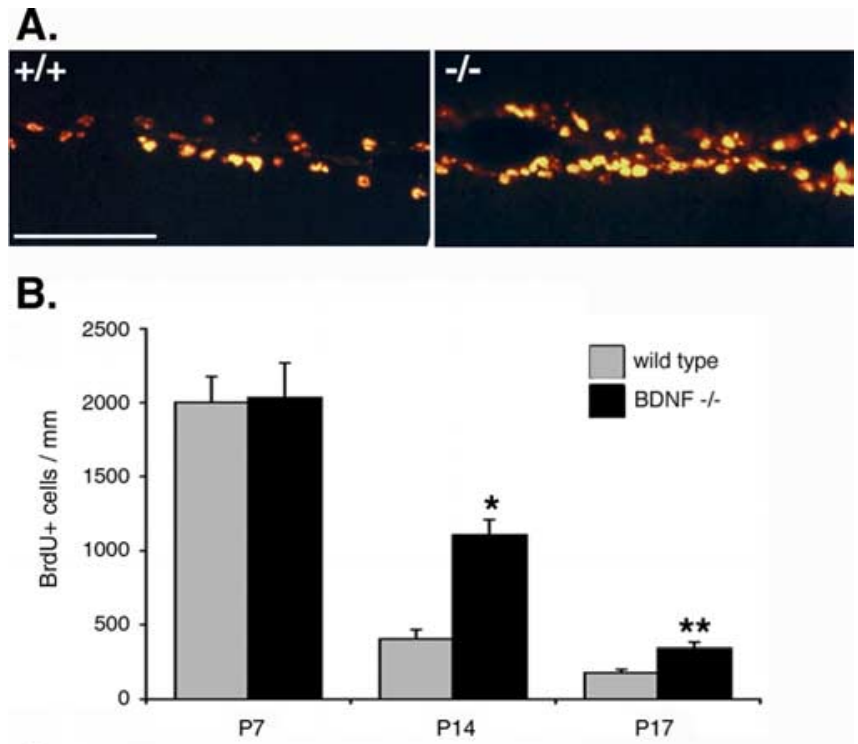

C.

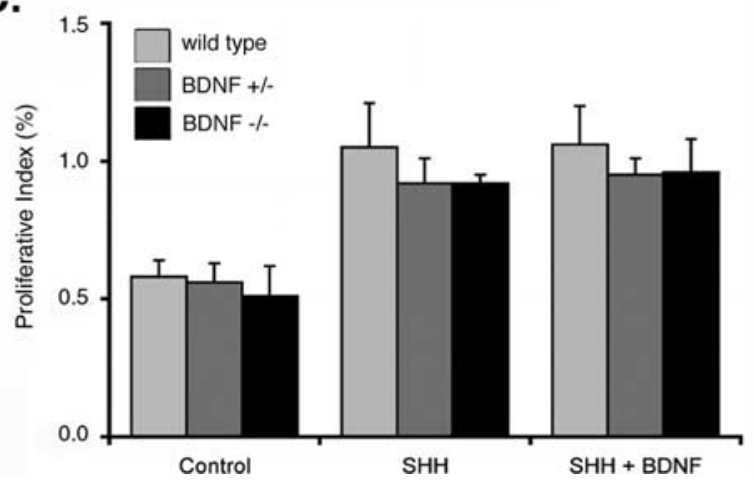

Figure 1. Granule cell precursors continue to proliferate when they remain in the EGL. $A, B$, Persistent proliferation in the EGL of BDNF-/- mice. Dividing granule cell precursors in wildtype and BDNF-/- littermates are labeled by systemic injection with $50 \mathrm{mg} / \mathrm{kg}$ BrdU. Cerebellar tissue is collected and processed for BrdU immunohistochemistry at $6 \mathrm{~h}$ after injection. $\boldsymbol{A}$, More cells incorporate BrdU in the BDNF-/- EGL compared with the wild-type EGL at P14. Representative pictures show immunofluorescent staining using antibody against BrdU. Scale bar, $50 \mu \mathrm{m}$. B, Quantification of proliferative index. Labeled cells are counted in nonadjacent midsagittal sections in two regions of the cerebellum, on folium 6 in the primary fissure, and on folium 9 in the secondary fissure. The proliferative index is calculated by dividing the number of $\mathrm{BrdU}+$ cells by the number of total cells (DAPI nuclear staining). The rate of proliferation in the $B D N F-/-E G L$ and wild-type EGL is equal at P7. The rate of proliferation in the mutant compared with the wild type is 2.7-fold higher atP14 and 1.9-fold higher at P17. ${ }^{*}<0.005$; ${ }^{* *} p<$ 0.05 (Student's $t$ test). C, BDNF does not directly affect granule cell proliferation. Dissociated granule cells from wild-type $(n=5), B D N F+/-(n=5)$, and BDNF- $-(n=4)$ cerebella are cultured for $48 \mathrm{~h}$ in the presence of $\mathrm{SHH}$ or both $\mathrm{SHH}$ and BDNF, with BrdU in the last $4 \mathrm{~h}$. The proliferative index is calculated by dividing the number of BrdU + cells by the number of total cells (DAPI nuclear staining). The response of BDNF- - granule cells does not differ from wild-type or BDNF+/- granule cells. Exogenous BDNF does not affect SHH-induced proliferation. Error bars represent SEM.

halted in the ML in the p35 mutant rather than in the EGL as occurs in the BDNF mutant (Fig. 2 B). To determine whether this impaired migration in the ML is accompanied by persistent proliferation, we labeled $p 35-/-$ and wild-type granule cell precursors by systemic injection with BrdU at P14 or P17 and processed the cerebella for immunohistochemistry $6 \mathrm{~h}$ after injection. In contrast to the results seen in $B D N F-/-$ mice, there is no difference in granule cell precursor proliferation between $p 35-/-$ and wild-type animals (Fig. 2C). Thus, mutation in BDNF blocks the initiation of granule cell precursor migration out of the EGL and leads to persistent proliferation, whereas mutation in $\mathrm{p} 35$, which 
A.

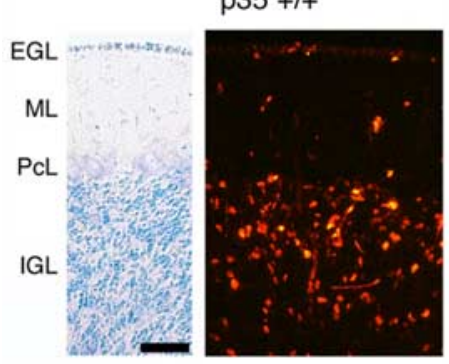

B.

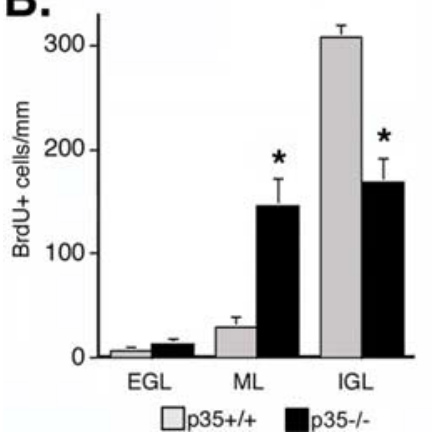

p35 \%-

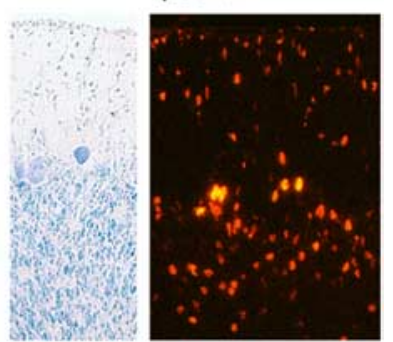

C.

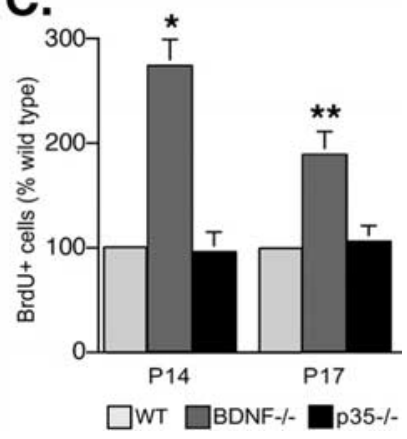

Figure 2. Granule cell proliferation is not increased in $p 35-/-$ mice. $\boldsymbol{A}, \boldsymbol{B}$, Impaired migration in the p35 mutant. Dividing granule cell precursors in the EGL in P11 wild-type and p35- / - littermates are labeled by systemic injection with $50 \mathrm{mg} / \mathrm{kg} \mathrm{BrdU}$. Cerebellar tissue is collected and processed for BrdU immunohistochemistry at $96 \mathrm{~h}$ after injection. $\boldsymbol{A}$, Representative pictures of wild-type versus $p 35-/-$ cerebellum at $96 \mathrm{~h}$ after injection. Nearly all BrdU+ wild-type granule cells have migrated into the IGL, whereas numerous labeled cells remain in the ML of $p 35-/-$ cerebellum. Adjacent sections stained with cresyl violet are provided to the left of each image. Scale bar, $50 \mu \mathrm{m}$. B, Quantification of impaired migration. Labeled cells are counted in nonadjacent midsagittal sections in two regions of the cerebellum, on folium 6 in the primary fissure, and on folium 9 in the secondary fissure. In the $p 35-/-$ cerebellum $(n=3)$ compared with the wild-type cerebellum $(n=3)$, more cells are in the ML and less cells are in the IGL at $96 \mathrm{~h}$ after injection. ${ }^{*} p<0.05$ (Student's $t$ test). C, Impaired migration in $p 35-1-$ does not lead to persistent proliferation. Dividing granule cell precursors in P14 or P17 mutant $(n=4)$ and wild-type (WT) $(n=4)$ littermates are labeled by systemic injection with $50 \mathrm{mg} / \mathrm{kg}$ BrdU; $6 \mathrm{~h}$ later, tissue is collected and processed for BrdU immunohistochemistry. Labeled cells are counted in nonadjacent midsagittal sections in on folium 6 in the primary fissure and on folium 9 in the secondary fissure. Compared with wild type, granule cell proliferation is increased in the EGL of BDNF-/-, but not $p 35-/-$, mice. Error bars represent SEM.

blocks the continued movement of these cells through the ML, does not affect proliferation. These data suggest that impaired migration does not inevitably promote proliferation.

\section{Granule cell precursors from a homogeneous population proliferate preferentially in the EGL}

Based on the in vivo evidence that persistent exposure of granule cell precursors to the environment of the EGL leads to proliferation, we asked whether the EGL is a specialized mitogenic niche for granule cell precursors. We developed an assay to directly compare the mitogenic effect of the EGL versus other cerebellar layers. In this assay, we purify P6 granule cell precursors and place a single-cell suspension of purified precursors in all layers of an organotypic cerebellar slice (supplemental Fig. 1, available at www. jneurosci.org as supplemental material) and use BrdU labeling to assess the proliferative response of introduced precursors. To facilitate the identification of introduced precursors, the cells are purified from GFP transgenic mice and introduced onto organotypic cerebellar slices taken from wild-type littermates, and the slices are stained with antibodies against GFP and BrdU (Fig. 3A). As shown, introduced granule cell precursors incorporate well

into the slice and resemble endogenous granule cells morphologically, with small round cell bodies and long processes that are often parallel or perpendicular to the pial surface (Fig. $3 B$ ).

Data analysis consists of cataloging every introduced GFP+ cell for location and for BrdU immunostaining. The outer EGL is easily distinguishable by BrdU labeling of endogenous granule cell precursors in the slice and is referred to as EGL. The inner EGL and the ML are often difficult to distinguish from each other and thus are included together as ML. The Purkinje cell layer $(\mathrm{PcL})$ can be distinguished by the autofluorescence in this layer adjacent to the BrdU-labeled endogenous Bergmann glia. The IGL and white matter (WM) can be distinguished readily by cell density difference in the bright-field picture. Using these consistent criteria, we count the number of GFP + and GFPBrdU double-positive cells in each layer. This system allows us to determine the proliferative response of introduced precursors that have been exposed to microenvironments of distinct layers.

We find that introduced granule cell precursors incorporate randomly in all layers but proliferate preferentially in the EGL. The distribution of GFP + cells over all layers of the cerebellum is random (Fig. 3C, green bars) (i.e., the percentage of GFP + found in a given layer corresponds to the percentage of its area per total area) (Altman and Bayer, 1997). However, the distribution of GFP-BrdU double-positive cells is not random; GFP-BrdU double-positive cells are preferentially located in the EGL compared with the other layers (Fig. 3C). The WM is another microenvironment in which we observe proliferation of introduced precursors (Fig. 3C; supplemental Fig. 2, available at www.jneurosci.org as supplemental material). This is consistent with recent data indicating that the WM is a location in which cerebellar stem cells proliferate at this age (Lee et al., 2005). The proliferative index in the PcL is highly variable because of the small number of cells in this layer; only $2 \%$ of introduced precursors are located in the PcL (Fig. 3C). Thus, in all subsequent analysis, we concentrate on the two granule cell layers, EGL and IGL, which are the biologically relevant locations for granule cells in normal development. A comparison of proliferative indices in the EGL and in the IGL shows that the introduced precursors proliferate at a rate threefold higher in the EGL (Fig. 3D). These data indicate that granule cell precursors from a homogeneous population proliferate preferentially in the EGL.

Two explanations could account for the observed distribution of proliferating cells. Positive factors could promote proliferation in the EGL; alternatively, negative factors could inhibit proliferation in the IGL. To address the possibility that the preferential proliferation of introduced granule cell precursors may arise because of inhibitory factors in the IGL, we asked whether the IGL contains factors that preclude a response to a granule cell mitogen, $\mathrm{SHH}$. The same procedures were used to introduce GFP+ granule cell precursors onto an organotypic cerebellar slice, and the system was cultured in the presence of SHH $(3 \mu \mathrm{g} / \mathrm{ml})$. We find that $\mathrm{SHH}$ increases proliferation in all layers, with a 2.3 -fold increase in the EGL and a 2.7-fold increase in the IGL (Fig. 4). This result indicates that the IGL contains no dominant inhibitory factor that can counter $\mathrm{SHH}$-induced proliferation. As controls for this assay, purified GFP + granule cell precursors are maintained in dissociated cell culture in parallel with each overlay experiment. Dissociated, purified granule cell precursors proliferate at a low basal rate, much lower than on the slice (data not shown), and the rate of proliferation is increased by $\mathrm{SHH}$ treatment (Fig. 4C). Although these results strongly suggest that the IGL does not contain inhibitory factors that can override the 


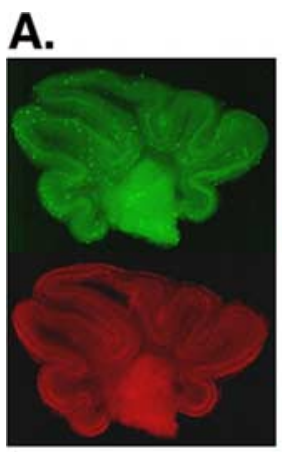

B.
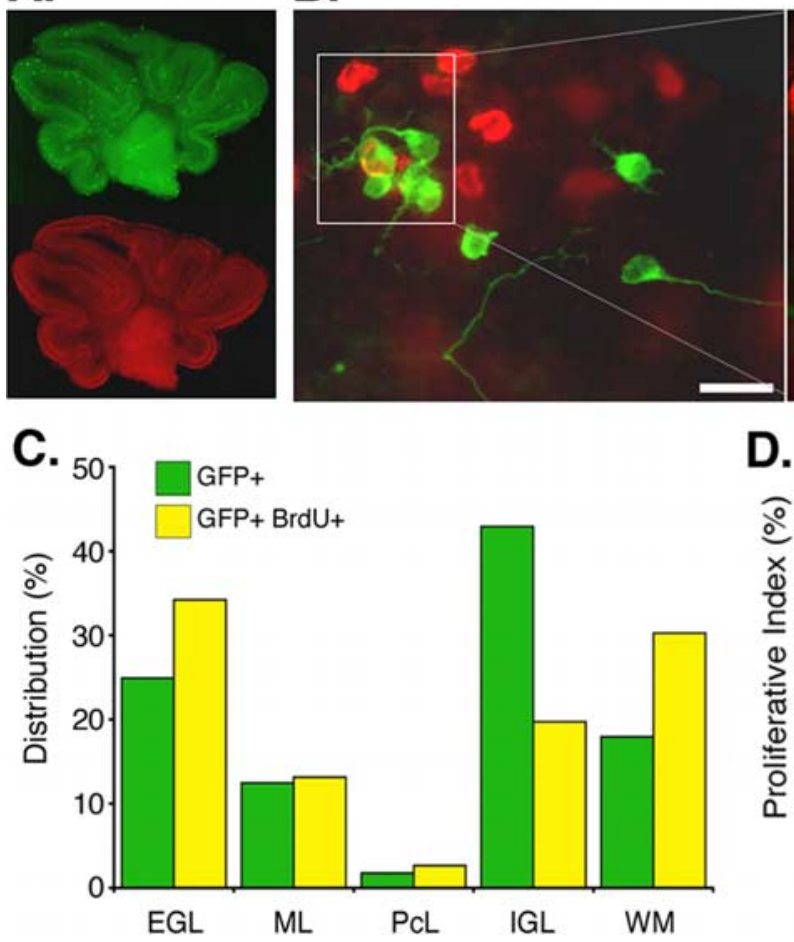

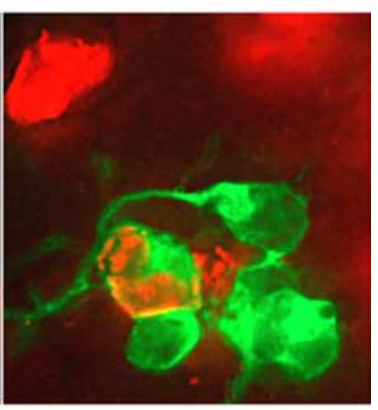

D.

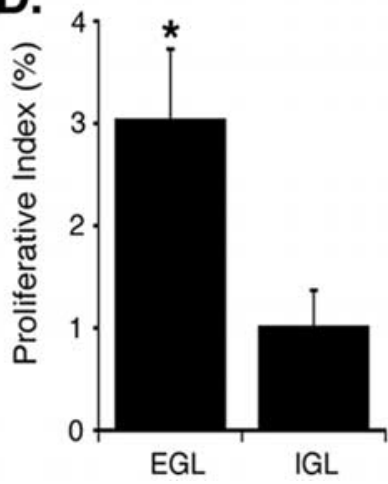

Figure 3. Introduced granule cell precursors proliferate preferentially in the EGL. $\boldsymbol{A}$, Introduced precursors are located randomly in all layers of the cerebellar slice. Representative pictures of an immunofluorescently stained slice are shown at low magnification. Green, GFP; red, BrdU. Thickness of the slice results in high background autofluorescence, which guides in layer identification. Scale bar, $1 \mathrm{~mm}$. $\boldsymbol{B}$, Representative picture of introduced granule cell precursors at high magnification. Granule cell precursors incorporate well into the organotypic slice, extend processes, and proliferate (as indicated by BrdU uptake). Green, GFP; red, BrdU. The higher magnification, optically sectioned image (from box) shows a representative GFP-BrdU double-positive cell in the EGL. Scale bar, $10 \mu \mathrm{m}$. C, The distribution of GFP+-introduced granule cell precursors is random, but the distribution of proliferating introduced precursors double positive for GFP and BrdU is not random. GFP + and GFP-BrdU double-positive cells are counted, and their location was cataloged (total cell number, 3500). The graph shows the distribution of all introduced precursors (green) and double-positive introduced precursors (yellow) across all layers of the cerebellum. Percentage distribution is calculated by dividing the number of GFP + precursors (or double-positive precursors) in a layer by the total number of GFP + precursors (or double-positive precursors). The green bars correlate well with the relative area of each layer: EGL, 24\%; ML, 12\%; PCL, 4\%; IGL, 36\%; WM, 24\% (Altman and Bayer, 1997). D, Introduced precursors proliferate preferentially in the EGL. The proliferative index is calculated by dividing the number of GFP-BrdU double-positive cells in a layer by the number of GFP + cells in the layer. The EGL has a proliferative index threefold higher than the IGL. ${ }^{*} p<0.05$ ( $n=8 ; 4$ experiments; Student's $t$ test). Error bars represent SEM.

mitogenic effect of $\mathrm{SHH}$, it is important to note that these studies do not exclude the possibility that factors in layers other than the outer EGL may help to inhibit proliferation. Together, these results indicate that a major reason that the introduced precursors proliferate preferentially in the EGL is that the EGL constitutes a mitogenic niche, which contains factors that promote proliferation and/or inhibit cell-cycle exit.

\section{Sonic Hedgehog is a critical component of the mitogenic niche in the EGL}

Because the above data demonstrate that the EGL constitutes a mitogenic niche that supports granule cell precursor proliferation, we investigated whether $\mathrm{SHH}$ is a critical component of this mitogenic niche. Of known mitogens for granule cell precursors, $\mathrm{SHH}$ is the most efficacious, and Purkinje cells express Shh mRNA, whereas granule cells express mRNAs encoding the Shh receptor, Ptc, and other downstream signaling components (Dahmane and Ruiz-i-Altaba, 1999; Wallace, 1999; WechslerReya and Scott, 1999). Thus, SHH is a likely component of the mitogenic niche in the EGL.
To study the function of endogenous $\mathrm{SHH}$ in the composition of the EGL, we used a $\mathrm{SHH}$-specific small molecule inhibitor, cyclopamine. Cyclopamine directly inhibits Smoothened, a positive effector of the $\mathrm{SHH}$ signaling pathway (Chen et al., 2002). The same procedures were used to introduce GFP + granule cell precursors into an organotypic cerebellar slice, and the system was cultured in the presence of $10 \mu \mathrm{M}$ cyclopamine. At this concentration, cyclopamine does not compromise the health of the slice or introduced precursors, and introduced precursors incorporate into the slice well (Fig. 5A). We could therefore use cyclopamine in this assay to determine whether $\mathrm{SHH}$ is a critical component of the mitogenic niche in the EGL. As shown, cyclopamine reduces proliferation of the introduced precursors. The cumulative rate of proliferation of introduced precursors is lower in cyclopaminetreated slices than in control slices. When we analyze proliferation in individual layers, we find that cyclopamine decreases proliferation in the EGL but does not decrease proliferation in the IGL (Fig. 5B). Thus, treatment with cyclopamine eliminates the preferential proliferation in the EGL. The selective decrease in proliferation observed in the EGL indicates that $\mathrm{SHH}$ is an important component of the mitogenic niche that is spatially restricted to the EGL.

To determine whether cyclopamine is blocking the response to $\mathrm{SHH}$ protein that is provided by the slice, we compared the effect of cyclopamine on precursors in purified culture and on introduced precursors in the slice. We treated high-density cultures of purified granule cell precursors with $10 \mu \mathrm{M}$ cyclopamine. Compared with the threefold or $76.5 \%$ decrease seen in the EGL, cyclopamine only decreases proliferation in purified culture by $11.7 \%$ (Fig. $5 C$ ). This result shows that the introduced precursors in the EGL proliferate because of SHH protein that is present in the EGL of the organotypic slice.

We therefore asked whether the mitogenic niche in the EGL is the location with the highest level of SHH protein. Consistent with previous studies (Gritli-Linde et al., 2002; Lewis et al., 2004), we observe $\mathrm{SHH}$ protein expression predominantly in the Purkinje cell bodies and dendrites, with lower and indistinguishable levels of protein in EGL and IGL (Fig. 6A). Thus, although SHH is critical for the mitogenic niche, the location of $\mathrm{SHH}$ protein alone does not account for its mitogenic effect.

This result led us to ask whether the EGL is the only location in which $\mathrm{SHH}$ is active. We performed an in situ hybridization experiment to examine expression of Shh target genes Gli1, Ptc1, and Hip1. Consistent with previous studies (Dahmane and Ruiz-i-Altaba, 1999; Wallace, 1999; Wechsler-Reya and Scott, 1999), Shh target genes are expressed in both granule cell layers, indicating that $\mathrm{SHH}$ is active in both layers. However, the expression patterns of the Shh target genes Gli1, Ptc, and Hip1 are 
A.

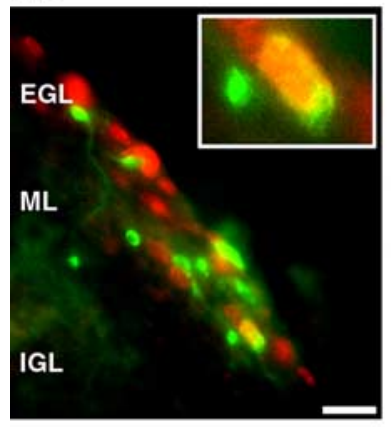

B.

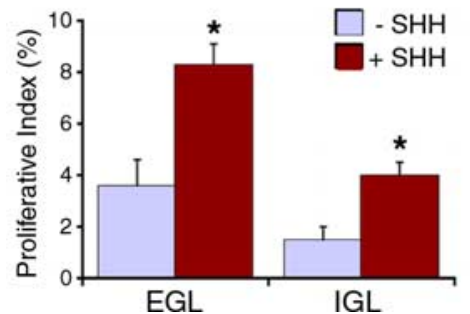

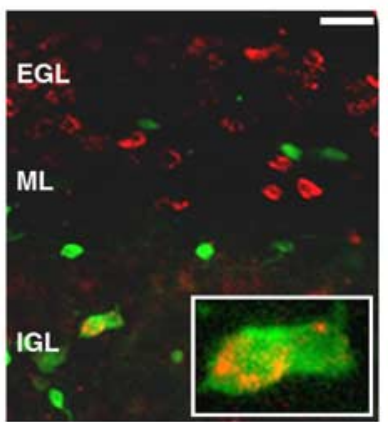

C.

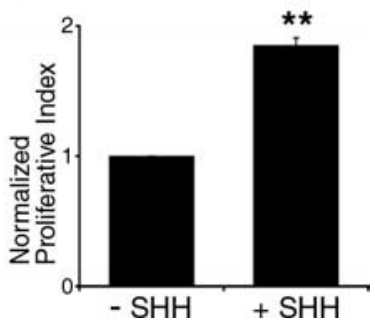

Figure 4. The IGL does not contain negative factors that can inhibit SHH-induced proliferation. Slices with introduced precursors are cultured in the presence of SHH ( $3 \mu \mathrm{g} / \mathrm{ml})$. A, Representative pictures of GFP-BrdU double-positive cells in the EGL and in the IGL. Green, GFP; red, BrdU. Scale bar, $10 \mu \mathrm{m}$. $\boldsymbol{B}$, Exogenous SHH increases the rate of proliferation of introduced precursors both in the EGL and the IGL. The proliferative index is calculated by dividing the number of GFP-BrdU double-positive cells in a layer by the number of GFP + cells in the layer. Adding $\mathrm{SHH}$ results in a 2.3-fold increase in the proliferative index in the EGL and a 2.7-fold increase in the IGL. ${ }^{*} p<0.05$ ( $n=5 ; 3$ experiments; Student's $t$ test). C, Purified granule cell precursors in parallel cultures also show increased proliferative index in response to $\mathrm{SHH} .{ }^{* *} p<$ 0.001 ( $n=5$; Student's $t$ test). Error bars represent SEM.

A.

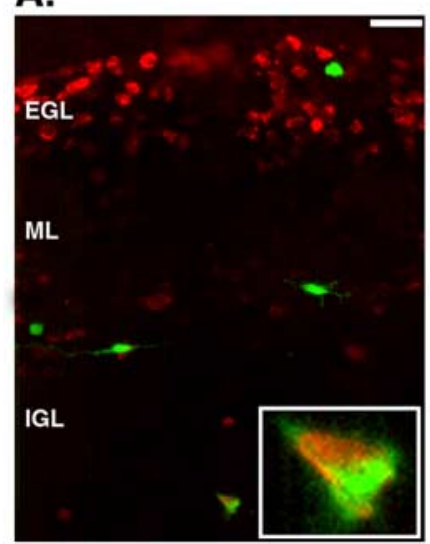

B.

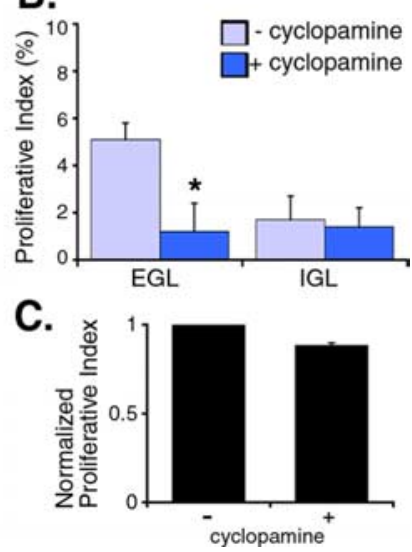

Figure 5. The EGL mitogenic niche requires endogenous SHH. Slices with introduced precursors are cultured in the presence of cyclopamine $(10 \mu \mathrm{M})$. $\boldsymbol{A}$, Representative picture of a GFPBrdU double-positive cell in the IGL. Green, GFP; red, BrdU. Scale bar, $10 \mu \mathrm{m}$. B, Blocking SHH function with cyclopamine decreases the rate of proliferation only in the EGL. The proliferative index is calculated by dividing the number of GFP-BrdU double-positive cells in a layer by the number of GFP + cells in the layer. Cyclopamine treatment results in a 76.5\% decrease in the proliferative index in the EGL. ${ }^{*} p<0.01$ ( $n=4 ; 4$ experiments; Student's $t$ test). $C$, In purified granule cell precursor cultures, cyclopamine has a mild inhibitory effect on proliferation: $11.7 \%$ decrease ( $p<0.05 ; n=4$; Student's $t$ test). Error bars represent SEM.

different in the EGL and IGL. The expression of the transcription factor Gli1 is at a higher level in the EGL than in the IGL. This transcription factor has been implicated previously in proliferative responses to Shh (Ruiz i Altaba, 1999). In contrast, expres-

sion of the receptor Ptc is higher in the IGL than in the EGL. The third target gene, Hip1, is expressed at the same level in the two granule cell layers (Fig. 6B). Thus, although $\mathrm{SHH}$ protein is present and active in both granule cell layers, the nature of the response to SHH differs. Positive or negative layer-selective modifiers of $\mathrm{SHH}$ activity may explain the difference between the mitogenic effects of distinct layers.

Some soluble growth factors are capable of modulating $\mathrm{SHH}-$ induced proliferation and could contribute to the mitogenic niche in the EGL. The chemokine CXCL12 has been shown to increase $\mathrm{SHH}$-induced proliferation of granule cell precursors in vitro (Klein et al., 2001). CXCL12 is expressed by the pia, which flanks the EGL, and granule cell precursors express its receptor, CXCR4. However, in our system, the pia is partially disrupted during the preparation of organotypic slices. We see no evidence that blocking CXCR4 function with the CXCR4-specific small molecule inhibitor AMD 3100 inhibits the mitogenic effect of the EGL (supplemental table, available at www.jneurosci.org as supplemental material). Thus, although CXCL12 may contribute to proliferation in vivo, factors that are made by the cerebellar parenchyma must also synergize with $\mathrm{SHH}$ to contribute to the mitogenic niche in the EGL.

\section{Discussion}

Migration away from a mitogenic niche promotes cell-cycle exit

Transition of precursors from a proliferative to a migratory state is a critical stage in neural development, but little is known about how this process is regulated. Here, we ask whether migration of precursors away from a mitogenic niche contributes to this transition. Using in vivo and in vitro approaches, we show that the EGL provides a mitogenic niche for granule cell precursors.

When granule cell precursors remain in the EGL in vivo because of impaired migration, they continue to proliferate. In the $B D N F-/-$ mice, increased proliferation is observed in the EGL at late stages of cerebellar development (P14 and P17), although there is no increase in proliferation at an earlier time (P7) (Fig. $1 B)$. Similarly, a mutation of the orphan receptor rev-erb $\alpha$, which impairs migration such that granule cell precursors are forced to remain in the EGL, also results in persistent proliferation of granule cell precursors at late stages of development (Chomez et al., 2000). Mutations in the gene encoding the astrotactin adhesion molecule also result in slow migration and a persistent EGL (Adams et al., 2002). In these mice, no difference in proliferation is observed in P6 astrotactin mutants, but proliferation was not assessed at later time points. Therefore, it is not yet clear whether the astrotactin mutant provides a third example of a mutation in which impaired migration results in persistent proliferation at later stages. The persistent proliferation seen in these cases is not an inevitable result of impaired migration, because granule cell precursors whose migration is halted in the ML instead of the EGL do not continue to proliferate. These data suggest that prolonged exposure to the microenvironment of the EGL results in persistent proliferation.

The Ptc mice provide additional genetic evidence that the EGL constitutes a proliferative microenvironment. In the $P t c+/-$ mouse, rests of granule cell precursors are observed on the surface of the cerebellum at P15. Cells in these rests exhibit characteristics of normal granule cell precursors in a younger EGL, and proliferation is increased in the rests compared with the P15 EGL (Kim et al., 2003). Although the mechanism by which these rests are formed is unknown, these data further support the hypothesis 

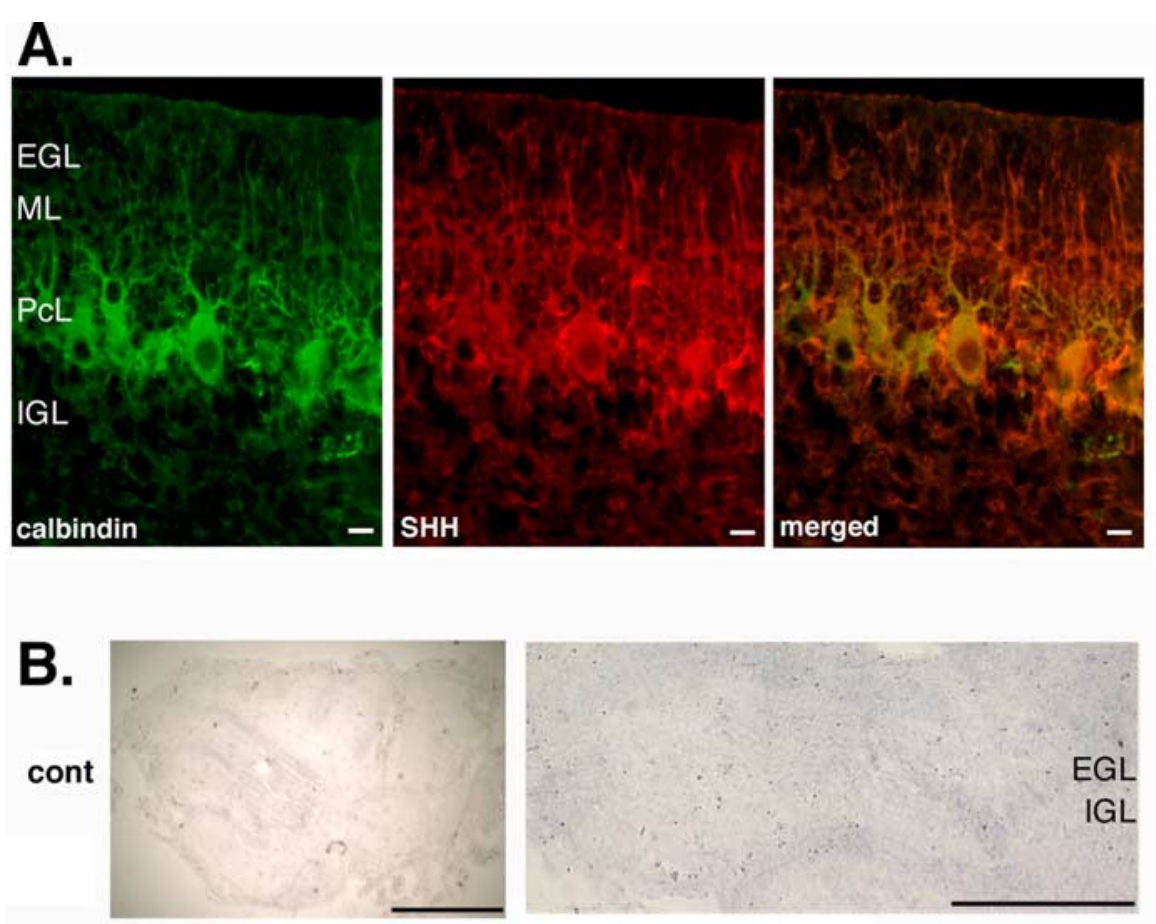

gli1
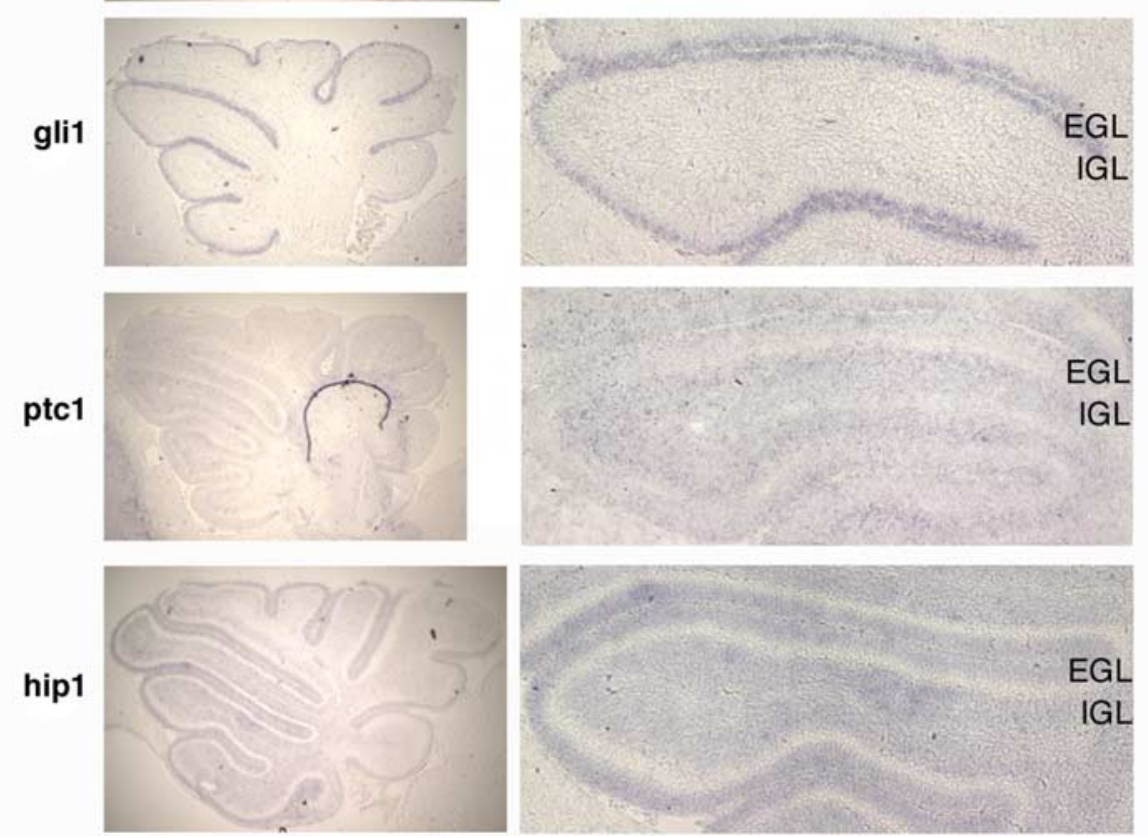

Figure 6. SHH protein is expressed in all layers, but different SHH response genes are expressed in EGL and IGL. $A_{\text {, Cryosections }}$ (5 $\mu \mathrm{m}$ thick) from P6 C57BL/6J are immunostained using calbindin antibody and SHH antibody. Green, Calbindin; red, SHH. Scale bar, $10 \mu \mathrm{m}$. B, Cryosections ( $14 \mu \mathrm{m}$ thick) from P6 C57BL/6J cerebella are hybridized with DIG-labeled RNA probes and visualized using anti-DIG antibody. Nonsense probe was used as negative control (cont). Scale bars: first column, $1 \mathrm{~mm}$; second column, 0.5 $\mathrm{mm}$.

that granule cell precursors continue to proliferate when they are exposed to the EGL microenvironment.

The in vivo evidence that the EGL is a critical mitogenic environment is corroborated by results from an in vitro assay using intact microenvironments in organotypic slice culture. We find that granule cell precursors exposed to the microenvironment of the EGL proliferate preferentially when compared with precursors exposed to other cerebellar microenvironments. Together, these results indicate that the EGL functions as a mitogenic niche and that migration out of this microenvironment stimulates precursors to exit the cell cycle.
Recent studies highlight the importance of mitogenic niches in supporting proliferation of precursors and maintaining self-renewing stem cells in many organ systems (Moore and Lemischka, 2004; Nishimura et al., 2005). The assay we use here uses a homogeneous population of precursor cells and intact microenvironments to directly identify a mitogenic niche in the developing cerebellum. By testing the ability of distinct microenvironments to stimulate proliferation of a homogeneous population of cells, this assay allows a direct comparison of microenvironments and eliminates cell-autonomous influences. An advantage of this approach is that we can assay the microenvironment as a whole rather than focusing on single components. Even without knowing all of the mitogenic components of the EGL, we can recognize it as a mitogenic niche. This assay may also be useful in other contexts to assess the effect of microenvironments on stem cell, precursor, or tumor cell proliferation.

It is important to note that the identification of the EGL as a mitogenic niche does not preclude a role for cell autonomous factors in cell-cycle exit. Previous studies provide evidence that cell autonomous changes are also critical for cessation of proliferation. For example, expression of cyclin-dependent kinase inhibitors such as p27/kinase inhibitor protein 1 (Kip1) terminates proliferation of granule cell precursors (Durand et al., 1998; Miyazawa et al., 2000). Cell-autonomous and noncell-autonomous mechanisms may interact in such a way that expression of p27/ Kip1 is inhibited by components of the mitogenic niche in the EGL, and migration of granule cells away from the EGL may allow the expression of this cellautonomous antiproliferative factor. It is also possible that cell-autonomous and non-cell-autonomous mechanisms work in parallel to terminate proliferation and achieve spatial restriction of precursor proliferation.

\section{Sonic Hedgehog is a critical component of the mitogenic niche}

The results reported here identify $\mathrm{SHH}$ as a critical component of the mitogenic niche in the EGL. The requirement for $\mathrm{SHH}$ in the localized proliferative microenvironment is demonstrated by blocking SHH signaling with the small molecule inhibitor cyclopamine. In the presence of cyclopamine, the proliferative rate of granule cell precursors exposed to the EGL is decreased to the same level as the proliferative rate of precursors exposed to the IGL. Thus, SHH signaling is required for the selective mitogenic effect of the EGL. This is consistent with previous studies demonstrating the importance of $\mathrm{SHH}$ for granule cell precursor proliferation (Dahmane and Ruiz-iAltaba, 1999; Wechsler-Reya and Scott, 1999; Lewis et al., 2004). 
Although the EGL contains endogenous $\mathrm{SHH}$, our immunohistochemical data indicate that $\mathrm{SHH}$ protein is present not only in the EGL but in all layers of the developing cerebellum. Furthermore, we find that distinct Shh target genes are preferentially expressed in the EGL (Gli1 and Hip1) or in the IGL (Ptc1 and Hip1), suggesting that $\mathrm{SHH}$ in both locations can induce the canonical Shh signaling pathways. One possible explanation to reconcile these data is that the EGL has the optimal concentration of SHH to induce Gli1 expression and proliferation and that the SHH concentration in the IGL, higher or lower than in the EGL, induces Ptcl expression and prevents proliferation. However, data that exogenous $\mathrm{SHH}$ further increases the rate of proliferation in all layers, including the EGL and the IGL, indicate this is not a likely explanation. Thus, we favor the hypothesis that modifiers influence the nature of the response to $\mathrm{SHH}$.

Both positive modifiers of $\mathrm{SHH}$-induced proliferation in the outer EGL and negative modifiers in other layers may contribute to the spatial restriction of the mitogenic niche. Positive modifiers of SHH detected in our assay may represent cell-surface or ECM molecules. Granule cell precursors, which make up a vast majority of the cellular environment of the EGL, express the cellsurface molecule Delta, which can contribute to precursor cell proliferation through the Notch signaling pathway (Solecki et al., 2001). ECM components, produced by granule cell precursors or by surrounding cells, can also modify the proliferative response to $\mathrm{SHH}$. Heparan sulfate proteoglycans (HSPGs) directly interact with $\mathrm{SHH}$ and modify the proliferative response of granule cell precursors (Rubin et al., 2002), and the interaction of SHH with laminin in the EGL may increase SHH downstream signaling (Pons et al., 2001). Thus, spatial and temporal regulation of ECM components is likely to play a critical role in modifying the $\mathrm{SHH}$ induced proliferative response. One way to test whether membrane-bound molecules such as HSPGs contribute to the mitogenic niche in the EGL is to introduce precursors onto slices that have been treated with fixative or heparinase. However, we find that fixation does not allow introduced precursors to adhere to the slice (data not shown). In the future, it will be important to use genetic methods for disrupting the SHH-HSPG interaction and monitor any resultant changes in the mitogenic niche in the EGL.

Although it is likely that there are positive modifiers of SHHinduced proliferation in the outer EGL, it is also possible that negative modifiers in other layers contribute to limiting $\mathrm{SHH}$ induced proliferation to the outer EGL. Some factors that have been shown to inhibit $\mathrm{SHH}$-induced proliferation in vitro include soluble factors such as the pituitary adenylate cyclase-activating polypeptide (Nicot et al., 2002), basic fibroblast growth factor (Wechsler-Reya and Scott, 1999), bone morphogenetic protein (Rios et al., 2004), and the ECM molecule vitronectin (Pons et al., 2001). In the future, the slice overlay assay can be used to identify both positive and negative modifiers of $\mathrm{SHH}$-induced proliferation.

Here, we used a novel approach, using a homogeneous population of precursor cells and intact microenvironments, to test directly for a mitogenic niche in the developing cerebellum. We find that the EGL provides a distinct microenvironment that supports granule cell precursor proliferation and that $\mathrm{SHH}$ is a critical component of this mitogenic niche in the EGL. Although $\mathrm{SHH}$ is a critical component, the mitogenic niche likely arises from synergistic interactions of multiple components. Future studies to define the precise composition of the cerebellar mitogenic niche will provide insight into the spatial and temporal regulation of proliferation in the developing nervous system. Synergistic actions of soluble factors and various cell-surface and
ECM molecules could provide a general mechanism that determines composition of various mitogenic niches.

\section{References}

Adams NC, Tomoda T, Cooper M, Dietz G, Hatten ME (2002) Mice that lack astrotactin have slowed neuronal migration. Development 129:965-972.

Altman J, Bayer SA (1997) Development of the cerebellar system: in relation to its evolution structure and functions, Ed 1, p 326. New York: CRC.

Borghesani PR, Peyrin JM, Klein R, Rubin J, Carter AR, Schwartz PM, Luster A, Corfas G, Segal RA (2002) BDNF stimulates migration of cerebellar granule cells. Development 129:1435-1442.

Chae T, Kwon YT, Bronson R, Dikkes P, Li E, Tsai LH (1997) Mice lacking p35, a neuronal specific activator of Cdk5, display cortical lamination defects, seizures, and adult lethality. Neuron 18:29-42.

Chen JK, Taipale J, Cooper MK, Beachy PA (2002) Inhibition of Hedgehog signaling by direct binding of cyclopamine to Smoothened. Genes Dev $16: 2743-2748$.

Chomez P, Neveu I, Mansen A, Kiesler E, Larsson L, Vennstrom B, Arenas E (2000) Increased cell death and delayed development in the cerebellum of mice lacking the rev-erbA(alpha) orphan receptor. Development 127:1489-1498.

Dahmane N, Ruiz-i-Altaba A (1999) Sonic hedgehog regulates the growth and patterning of the cerebellum. Development 126:3089-3100.

Durand B, Fero ML, Roberts JM, Raff MC (1998) p27Kip1 alters the response of cells to mitogen and is part of a cell-intrinsic timer that arrests the cell cycle and initiates differentiation. Curr Biol 8:431-440.

Ernfors P, Lee KF, Jaenisch R (1994) Mice lacking brain-derived neurotrophic factor develop with sensory deficits. Nature 368:147-150.

Flint AC, Kriegstein AR (1997) Mechanisms underlying neuronal migration disorders and epilepsy. Curr Opin Neurol 10:92-97.

Gao W, Zheng J, Karihaloo M (1995) Neurotrophin-4/5 (NT-4/5) and brain-derived neurotrophic factor (BDNF) act at later stages of cerebellar granule cell differentiation. J Neurosci 15:2656-2667.

Gleeson JG (2000) Classical lissencephaly and double cortex (subcortical band heterotopia): LIS1 and doublecortin. Curr Opin Neurol 13:121-125.

Gritli-Linde A, Bei M, Maas R, Zhang XM, Linde A, McMahon AP (2002) Shh signaling within the dental epithelium is necessary for cell proliferation, growth and polarization. Development 129:5323-5337.

Im SH, Chung CK, Kim SK, Cho BK, Kim MK, Chi JG (2004) Pleomorphic xanthoastrocytoma: a developmental glioneuronal tumor with prominent glioproliferative changes. J Neurooncol 66:17-27.

Kim JY, Nelson AL, Algon SA, Graves O, Sturla LM, Goumnerova LC, Rowitch DH, Segal RA, Pomeroy SL (2003) Medulloblastoma tumorigenesis diverges from cerebellar granule cell differentiation in patched heterozygous mice. Dev Biol 263:50-66.

Klein RS, Rubin JB, Gibson HD, DeHaan EN, Alvarez-Hernandez X, Segal RA, Luster AD (2001) SDF-1 alpha induces chemotaxis and enhances Sonic hedgehog-induced proliferation of cerebellar granule cells. Development 128:1971-1981.

Kwon YT, Tsai LH (1998) A novel disruption of cortical development in p35(-/-) mice distinct from reeler. J Comp Neurol 395:510-522.

Lee A, Kessler JD, Read TA, Kaiser C, Corbeil D, Huttner WB, Johnson JE, Wechsler-Reya RJ (2005) Isolation of neural stem cells from the postnatal cerebellum. Nat Neurosci 8:723-729.

Lewis PM, Gritli-Linde A, Smeyne R, Kottmann A, McMahon AP (2004) Sonic hedgehog signaling is required for expansion of granule neuron precursors and patterning of the mouse cerebellum. Dev Biol 270:393-410.

Miyazawa K, Himi T, Garcia V, Yamagishi H, Sato S, Ishizaki Y (2000) A role for $\mathrm{p} 27 / \mathrm{Kip} 1$ in the control of cerebellar granule cell precursor proliferation. J Neurosci 20:5756-5763.

Moore KA, Lemischka IR (2004) “Tie-ing” down the hematopoietic niche. Cell 118:139-140.

Nicot A, Lelievre V, Tam J, Waschek JA, DiCicco-Bloom E (2002) Pituitary adenylate cyclase-activating polypeptide and sonic hedgehog interact to control cerebellar granule precursor cell proliferation. J Neurosci 22:9244-9254.

Nishimura EK, Granter SR, Fisher DE (2005) Mechanisms of hair graying: 
incomplete melanocyte stem cell maintenance in the niche. Science 307:720-724.

Polleux F, Ghosh A (2002) The slice overlay assay: a versatile tool to study the influence of extracellular signals on neuronal development. Sci STKE 2002:PL9.

Polleux F, Giger RJ, Ginty DD, Kolodkin AL, Ghosh A (1998) Patterning of cortical efferent projections by semaphorin-neuropilin interactions. Science 282:1904-1906.

Pons S, Trejo JL, Martinez-Morales JR, Marti E (2001) Vitronectin regulates Sonic hedgehog activity during cerebellum development through CREB phosphorylation. Development 128:1481-1492.

Rios I, Alvarez-Rodriguez R, Marti E, Pons S (2004) Bmp2 antagonizes sonic hedgehog-mediated proliferation of cerebellar granule neurones through Smad5 signalling. Development 131:3159-3168.

Rubin JB, Choi Y, Segal RA (2002) Cerebellar proteoglycans regulate sonic hedgehog responses during development. Development 129:2223-2232.

Ruiz i Altaba A (1999) Gli proteins and Hedgehog signaling: development and cancer. Trends Genet 15:418-425.

Schwartz PM, Borghesani PR, Levy RL, Pomeroy SL, Segal RA (1997) Abnormal cerebellar development and foliation in $B D N F-/-$ mice reveals a role for neurotrophins in CNS patterning. Neuron 19:269-281.

Segal RA, Takahashi H, McKay RD (1992) Changes in neurotrophin re- sponsiveness during the development of cerebellar granule neurons. Neuron 9:1041-1052.

Solecki DJ, Liu XL, Tomoda T, Fang Y, Hatten ME (2001) Activated Notch2 signaling inhibits differentiation of cerebellar granule neuron precursors by maintaining proliferation. Neuron 31:557-568.

Stoppini L, Buchs PA, Muller D (1991) A simple method for organotypic cultures of nervous tissue. J Neurosci Methods 37:173-182.

Tanaka S, Sekino Y, Shirao T (2000) The effects of neurotrophin-3 and brain-derived neurotrophic factor on cerebellar granule cell movement and neurite extension in vitro. Neuroscience 97:727-734.

Tsai LH, Delalle I, Caviness Jr VS, Chae T, Harlow E (1994) p35 is a neuralspecific regulatory subunit of cyclin-dependent kinase 5 . Nature 371:419-423.

Wallace VA (1999) Purkinje-cell-derived Sonic hedgehog regulates granule neuron precursor cell proliferation in the developing mouse cerebellum. Curr Biol 9:445-448.

Waschek JA, Casillas RA, Nguyen TB, DiCicco-Bloom EM, Carpenter EM, Rodriguez WI (1998) Neural tube expression of pituitary adenylate cyclase-activating peptide (PACAP) and receptor: potential role in patterning and neurogenesis. Proc Natl Acad Sci USA 95:9602-9607.

Wechsler-Reya RJ, Scott MP (1999) Control of neuronal precursor proliferation in the cerebellum by Sonic Hedgehog. Neuron 22:103-114. 